Pacific Journal of Mathematics

ON THE NUMBER OF POLYNOMIALS OF AN IDEMPOTENT 


\title{
ON THE NUMBER OF POLYNOMIALS OF AN IDEMPOTENT ALGEBRA I
}

\author{
G. GRÄTZER AND J. PŁONKA
}

This paper deals with the number $p_{n}(\mathfrak{C})$ of essentially $n$ ary polynomials of an idempotent universal algebra $\mathfrak{A}$. Under the condition that there is a commutative binary polynomial $\cdot$ it is proved that $p_{n+1}(\mathfrak{U}) \geqq p_{n}(\mathfrak{U})+(n-1)$, provided $p_{n}(\mathfrak{U}) \neq 1$. If - is also associative this inequality is improved to

$$
p_{n+1}(\mathfrak{U}) \geqq p_{n}(\mathfrak{U})+1+\max \left\{p_{n}(\mathfrak{U}), n+1\right\} \text {. }
$$

A sequence $\mathfrak{p}=\left\langle p_{0}, p_{1}, \cdots\right\rangle$ is called representable (see [6]) if for some algebra $\mathfrak{A}, p_{n}=p_{n}(\mathfrak{U})$ for all $n \geqq 0$. The basic problem is the characterization of representable sequences. Earlier results on representability (see [5] and [6]) were of the type that sequences satisfying some very mild condition (e.g., $p_{0}>0$ ) are all representable, and so the $p_{i}$ are independent.

In this paper we make a first attack on the idempotent case $\left(p_{0}=p_{1}=0\right.$, in other words, $f(x, \cdots, x)=x$ for every operation $\left.f\right)$. We conjecture that for idempotent algebras the $p_{n}(\mathfrak{2})$ are not independent. In fact, we think that with one exception the sequence $\left\langle p_{n}(\mathfrak{U})\right\rangle$ is increasing from some $m$. Our general conjecture is the following:

Conjecture. Let $\mathfrak{A}$ be an idempotent algebra different from the idempotent reduct of a Boolean group. ${ }^{1}$ Then there exists an integer $m$ such that $1<p_{n}(\mathfrak{H})<\boldsymbol{\aleph}_{0}$ implies that $p_{n}(\mathfrak{H})<p_{n+1}(\mathfrak{U})$ for every $n>m$.

To verify this conjecture one should make use of K. Urbanik's [9] classification of idempotent algebras using the set

$$
Z(\mathfrak{U})=\left\{n \mid n \geqq 2, p_{n}(\mathfrak{X})=0\right\} \text {. }
$$

The structure of $\mathfrak{A}$ is quite well determined by $Z(\mathfrak{A})$ except if $Z(\mathfrak{A})=$ $\varnothing$, or $Z(\mathfrak{U})=\{2\}$. In this paper we take up part of the case $Z(\mathfrak{U})=$ $\varnothing$. If $Z(\mathfrak{U})=\varnothing$, then $p_{2}(\mathfrak{U}) \neq 0$, hence there exist binary polynomials; we shall discuss the case when there exist commutative binary polynomials.

THEOREM 1. Let $\mathfrak{A}$ be an idempotent algebra having a commutative binary polynomial. Then $p_{n}(\mathfrak{U}) \neq 1$ implies that

${ }^{1}$ Let $\langle G ;+\rangle$ be an abelian group; it is called Boolean if $2 x=0$ for all $x \in G$. The algebra $\langle G ; g\rangle$, where $g$ is a ternary operation defined by $g(x, y, z)=x+y+z$ is called the idempotent reduct of $\langle G ;+\rangle$. 


$$
p_{n+1}(\mathfrak{U}) \geqq p_{n}(\mathfrak{U})+(n-1) \text {. }
$$

The commutative binary polynomial that is assumed to exist is either associative or nonassociative. Accordingly, the proof of Theorem 1 splits into two completely different cases. In the nonassociative case one observes that for $n>2$ the assumption $p_{n}(\mathfrak{U}) \neq 1$ is superfluous (since $p_{3}(\mathfrak{H}) \geqq 3$ ). In the associative case we can prove a result that is much sharper:

THEOREm 2. Let $\mathfrak{A}$ be an idempotent algebra having a commutative and associative binary polynomial. Then $p_{n}(\mathfrak{H}) \neq 1(n \geqq 2)$ implies that

$$
p_{n+1}(\mathfrak{U}) \geqq p_{n}(\mathfrak{U})+1+\max \left\{p_{n}(\mathfrak{U}), n+1\right\} \text {. }
$$

The example given in $\S 2$ will show that the two inequalities making up (2) are sharp.

Many conclusion can be drawn from Theorems 1 and 2 .

Let us call a sequence $\left\langle p_{i}\right\rangle$ conditionally strictly increasing if $1<p_{i}<\aleph_{0}$ implies $p_{i}<p_{i+1}$.

CoRollary 1. Let $\mathfrak{A}$ be an idempotent algebra having a commutative and associative binary polynomial. If the sequence

$$
\left\langle p_{n}(\mathfrak{U}), p_{n+1}(\mathfrak{U}), \cdots\right\rangle
$$

is not conditionally strictly increasing for any $n \geqq 2$, then $\mathfrak{A}$ is equivalent to a semilattice.

Corollary 2. The only representable sequence $\left\langle 0,0, p_{2}, p_{3}, \cdots\right\rangle$ satisfying $p_{2}=1, p_{3} \leqq 2$ for which $\left\langle p_{n}, p_{n+1}, \cdots\right\rangle$ is not conditionally strictly increasing for any $n \geqq 2$ is $\langle 0,0,1, \cdots, 1, \cdots\rangle$.

The last condition of Corollary 2 is satisfied if the sequence $\left\langle p_{n}\right\rangle$ is assumed to be bounded. Under this assumption the conclusion of Corollary 2 is the same as the conclusion of the Theorem in [4] (however, the other assumptions in [4] are weaker than those in Corollary 2).

CoRollaRY 3. Let $\mathfrak{X}$ be a commutative idempotent groupoid (i.e., an algebra with a single binary operation). If $\mathfrak{A}$ is not equivalent to a semilattice, then for $n \geqq 3$

$$
p_{n}(\mathfrak{U}) \geqq \frac{(n-1)(n-2)}{2}+2 \text {. }
$$

Since $\mathfrak{A}$ is not equivalent to a semilattice the binary polynomial 
is not associative. Hence $p_{3}(\mathfrak{U}) \geqq 3$. Thus by (1):

$$
\begin{aligned}
p_{n}(\mathfrak{Q}) & \geqq p_{n-1}(\mathfrak{Q})+(n-2) \geqq \cdots \geqq(n-2)+\cdots+2+3 \\
& =\frac{(n-1)(n-2)}{2}+2 .
\end{aligned}
$$

A weaker result, namely $p_{n}(\mathfrak{A})>n$ was proved by J. Dudek [1]. A stronger result, namely

$$
p_{n}(\mathfrak{U}) \geqq \frac{1}{3}\left(2^{n}-(-1)^{n}\right)
$$

is proved in [3].

A rather unexpected application of Theorem 2 is given in [3].

For the notation and basic concepts used in this paper see [2].

In $\S 2$ we present some facts concerning binary operations. Constructions of $(n+1)$-ary polynomials from $n$-ary ones are given in $\S 3$. The inequality $p_{n+1} \geqq 2 p_{n}+1$ is proved in $\S 4$, while $p_{n+1} \geqq p_{n}+n+2$ is proved in $\S 5$, concluding the proof of Theorem 2. Finally, Theorem 1 is verified in $\S 6$.

2. Binary operations. Let us consider an algebra $\mathfrak{U}=\langle A ; \cdot, 0\rangle$ with two binary operations - and $\circ$ satisfying the following set of identities:

$$
\begin{gathered}
x \cdot x=x, x \cdot y=y \cdot x, x \cdot(y \cdot z)=(x \cdot y) \cdot z \\
x \circ x=x, x \circ(y \circ z)=(x \circ y) \circ z, x \circ(y \circ z)=x \circ(z \circ y) \\
(x \cdot y) \circ z=(x \circ z) \cdot(y \circ z), x \circ(y \cdot z)=(x \circ y) \cdot(x \circ z) \\
(x \cdot y) \circ x=x \cdot y,
\end{gathered}
$$

that is $\langle A ; \cdot\rangle$ is a semilattice and $\circ$ is a partition function in the sense of J. Płonka [8]. It follows from the identities (4) - (7) (and more directly from Theorem 1 of [8]) that for $n \geqq 2$ this algebra has exactly $2^{n}-1$ essentially $n$-ary polynomials. These can be described as follows: Let $\left\{x_{i_{0}}, \cdots, x_{i_{k}}\right\},\left\{x_{i_{k+1}}, \cdots, x_{i_{n-1}}\right\}$ be a partitioning of $\left\{x_{0}, \cdots, x_{n-1}\right\}$ into two nondisjoint sets; then

$$
\left(x_{i_{0}} \cdot x_{i_{1}} \cdot \cdots \cdot x_{i_{k}}\right) \circ\left(x_{i_{k+1}} \cdots \cdot x_{i_{n-1}}\right)
$$

is an essentially $n$-ary polynomial, and every essentially $n$-ary polynomial excepting $x_{0} \cdots \cdots \cdot x_{n-1}$ has a unique representation in this form, yielding $p_{n}(\mathfrak{U})=2^{n}-1$.

Since $2^{n+1}-1=2\left(2^{n}-1\right)+1$, the inequality $p_{n+1} \geqq 2 p_{n}+1$ cannot be improved. Also, for $n=2$ we get $p_{2}=3, p_{3}=7$, that is $p_{3}=p_{2}+$ $2+2$. Hence $p_{n+1} \geqq p_{n}+n+2$ cannot be sharpened to $p_{n+1} \geqq p_{n}+$ 
$n+k$ for any $k>2$.

All polynomials of the form (8) can be proved distinct under rather mild conditions:

LEMMA 1. Let $\langle A ; \cdot\rangle$ be a semilattice and let $\circ$ be an idempotent essentially binary operation which is noncommutative, and satisfies

$$
(x \cdot y) \circ z=x \cdot(y \circ z) \cdot
$$

Then all the polynomials given in (8) are distinct, essentially n-ary, and different from $x_{0} \cdot \cdots \cdot x_{n-1}$.

Proof. If (8) does not depend on $x_{i_{j}}$ then by symmetry, (8) does not depend on any variable in the same group. By identifying the variables in the same group we get that $x \circ y$ is not essentially binary. The first group of variables can be distinguished from the second by the fact that by (9) they can be brought outside. This cannot be done by any variable in the second group because it would imply the commutativity of $\circ$. This also shows that (8) is distinct from $x_{0} \cdot \ldots$ $\cdot x_{n-1}$, completing the proof of Lemma 1.

Another lemma we need deals with commutative binary operations.

LEMMA 2. Let - and + be distinct idempotent binary commutative operations, and let $\cdot$ be associative. Then the polynomials

$$
\begin{aligned}
& (x+y)+z,(y+z)+x,(z+x)+y,(x+y) \cdot z,(y+z) \cdot x \\
& (z+x) \cdot y,(x \cdot y)+z,(y \cdot z)+x,(z \cdot x)+y
\end{aligned}
$$

are all essentially ternary and at least seven of them are distinct. The polynomial $x \cdot y \cdot z$ cannot equal any one of these.

The proof is a straightforward combination of Lemmas 1-4 of [7], including the statements made in the proofs of the same.

3. Constructions of polynomials. In this section we deal with an idempotent algebra having a fixed binary commutative and associative polynomial :; for brevity, we sometimes write $x y$ for $x \cdot y$. Let $p$ be an $n$-ary polynomial. We define $n+1$ constructions: $M_{0}, \cdots$, $M_{n-1}$ and $S$ :

$$
\begin{gathered}
p M_{i}=p\left(x_{0}, \cdots, x_{i-1}, x_{i} \cdot x_{n}, \cdots, x_{n-1}\right) \\
p S=p \cdot x_{n} .
\end{gathered}
$$

Let $P_{n}$ denote the set of all essentially $n$-ary polynomials.

The next six lemmas describe the behaviour of the $M_{i}$ and of $S$. 
Lemma 3. $M_{i}$ is a one-to-one map of $P_{n}$ into $P_{n+1}$.

Proof. We prove the statement for $i=0$. Let $p \in P_{n}$. Then $p M_{0}=p\left(x_{0} x_{n}, x_{1}, \cdots, x_{n-1}\right)=q$. Since the substitution $x_{0}=x_{n}$ in $q$ yields $p$ we get immediately that (i) $M_{0}$ is one-to-one; (ii) $p M_{0}$ depends on $x_{1}, \cdots, x_{n-1}$, and on at least one of $x_{0}$ and $x_{n}$. Since $x_{0}$ and $x_{n}$ are symmetric in $q, q$ depends on both, completing the proof.

Lemma 4. $S$ is a one-to-one map of $P_{n}$ into $P_{n+1}$.

Proof. Let $p \in P_{n}$. Substituting $x_{0}=\cdots=x_{n-1}$ in $p S=p x_{n}$ we get $x_{0} x_{n}$ depending on $x_{0}$ and $x_{n}$; thus $p x_{n}$ depends on $x_{n}$. If $p x_{n}$ does not depend on $x_{i}(0 \leqq i<n)$, then $p\left(x_{0}, \cdots, x_{n-1}\right) \cdot p\left(y_{0}, \cdots, y_{n-1}\right)$ depends neither on $x_{i}$ nor on $y_{i}$ by the commutativity of $\cdot$, contradicting the fact that after the substitution $x_{j}=y_{j}, 0 \leqq j<n$, the polynomial depends on $x_{i}$. Now let $p, q \in P_{n}, p S=q S$, that is $p x_{n}=q x_{n}$. Substituting $x_{n}=p$, then $x_{n}=q$ we get

$$
p=p \cdot p=q \cdot p=p \cdot q=q \cdot q=q,
$$

completing the proof.

REMARK. Note that Lemmas 3 and 4 do not use the associativity of .. These lemmas are applied in these more general forms in [3].

Lemma 5. Let $i \neq j, p, q \in P_{n}$. Then $p M_{i}=q M_{j}$ implies $p=q$.

Proof. To simplify the notation let $i=0, j=1$. Then

$$
p\left(x_{0} x_{n}, x_{1}, \cdots, x_{n-1}\right)=q\left(x_{0}, x_{1} x_{n}, \cdots, x_{n-1}\right) .
$$

Compute:

$$
p\left(x_{0} y_{0}, x_{1} y_{1}, x_{2}, \cdots, x_{n-1}\right)=q\left(x_{0}, x_{1} y_{1} y_{0}, \cdots\right)=p\left(x_{0} x_{1}, y_{0} y_{1}, \cdots\right) .
$$

Hence

$$
p\left(x_{0}, x_{1}, \cdots\right)=p\left(x_{0} x_{1}, x_{0} x_{1}, \cdots\right)
$$

Similarly,

$$
q\left(x_{0}, x_{1}, \cdots\right)=q\left(x_{0} x_{1}, x_{0} x_{1}, \cdots\right) .
$$

Substituting $x_{0}, x_{1}$ and $x_{n}$ by $x_{0} x_{1}$ (13) yields

$$
\begin{aligned}
p\left(x_{0} x_{1}, x_{0} x_{1}, \cdots\right) & =p\left(x_{0} x_{1} \cdot x_{0} x_{1}, x_{0} x_{1}, \cdots\right) \\
& =q\left(x_{0} x_{1}, x_{0} x_{1} \cdot x_{0} x_{1}, \cdots\right)=q\left(x_{0} x_{1}, x_{0} x_{1}, \cdots\right) .
\end{aligned}
$$

(14)-(16) give $p=q$, as required. 
Lemma 6. Let $p, q \in P_{n}$. Then $p M_{i}=q S$ implies $p=q$.

Proof. To simplify the notation let $i=0$. Then

$$
p\left(x_{0} x_{n}, x_{1}, \cdots, x_{n-1}\right)=q\left(x_{0}, \cdots, x_{n-1}\right) x_{n} .
$$

Therefore,

$$
\begin{aligned}
q\left(x_{0} y, x_{1}, \cdots\right) x_{n} & =p\left(x_{0} x_{n} y, x_{1}, \cdots\right)=q\left(x_{0}, \cdots\right) x_{n} y \\
& =q(y, \cdots) x_{0} x_{n}=q\left(x_{0} x_{n}, \cdots\right) y .
\end{aligned}
$$

Now compute (applying (18) in every step):

$$
\begin{aligned}
q M_{0} & =q\left(x_{0} x_{n}, x_{1}, \cdots\right)=q\left(x_{0} x_{n}, \cdots\right) \cdot q\left(x_{0} x_{n}, \cdots\right) \\
& =q\left(x_{0} x_{n}, \cdots\right) \cdot q\left(x_{0} x_{n}, \cdots\right) x_{0}=q\left(x_{0} x_{n} \cdot q\left(x_{0} x_{n}, \cdots\right), \cdots\right) x_{0} \\
& =q\left(x_{0} x_{n} \cdot q\left(x_{0}, \cdots\right), \cdots\right) x_{0}=q\left(x_{0}, \cdots\right) \cdot q\left(x_{0}, \cdots\right) \cdot x_{0} x_{n} \\
& =q\left(x_{0}, \cdots\right) x_{n}=q S .
\end{aligned}
$$

Hence

$$
p M_{0}=q S=q M_{0},
$$

and so by Lemma 5 we conclude that $p=q$.

Lemma 7. Let $p, q \in P_{n}$, and $i \neq j$. Then $p M_{i}=p M_{j}$ if and only if

$$
\begin{aligned}
& p\left(x_{0}, \cdots, x_{i}, \cdots, x_{j}, \cdots, x_{n-1}\right) \\
= & p\left(x_{0}, \cdots, x_{i} x_{j}, \cdots, x_{i} x_{j}, \cdots, x_{n-1}\right) .
\end{aligned}
$$

Proof. Let $i=0, j=1$, and assume (19), that is,

$$
p=p\left(x_{0} x_{1}, x_{0} x_{1}, x_{2}, \cdots, x_{n-1}\right) \text {. }
$$

Then

$$
\begin{aligned}
p M_{0} & =p\left(x_{0} x_{n}, x_{1}, \cdots\right)={ }^{(20)} p\left(x_{0} x_{n} x_{1}, x_{0} x_{n} x_{1}, \cdots\right) \\
& ={ }^{(20)} p\left(x_{0}, x_{1} x_{n}, \cdots\right)=p M_{1} .
\end{aligned}
$$

Conversely, if $p M_{0}=p M_{1}$, then

$$
p\left(x_{0} x_{n}, x_{1}, \cdots\right)=p\left(x_{0}, x_{1} x_{n}, \cdots\right),
$$

and so

$$
\begin{aligned}
p\left(x_{0}, x_{1}, \cdots\right) & =p\left(x_{0} x_{0}, x_{1}, \cdots\right)={ }^{(20)} p\left(x_{0}, x_{0} x_{1}, \cdots\right) \\
& =p\left(x_{0},\left(x_{0} x_{1}\right) x_{1}, \cdots\right)=p\left(x_{0} x_{1}, x_{0} x_{1}, \cdots\right),
\end{aligned}
$$

completing the proof.

Finally, we introduce some notations that will be useful in the sequel, and using these we characterize semilattice polynomials.

For $p \in P_{n}$ let $G(p)$ denote the group of all permutations $\alpha$ of 
$\{0, \cdots, n-1\}$ satisfying

$$
p\left(x_{0}, \cdots, x_{n-1}\right)=p\left(x_{0 \alpha}, \cdots, x_{(n-1) \alpha}\right) .
$$

$G(p)$ is the symmetry group of $p$, and it is a subgroup of $S(n)$, the symmetric group on $n$ letters. Then

LEMma 8. The index of $G(p)$ in $S(n)$ is the same as the number of polynomials arising from $p$ by permuting the variables.

Proof is obvious.

For $\alpha \in S(n), p \in P_{n}$ define $p^{\alpha} \in P_{n}$ by

$$
p^{\alpha}\left(x_{0}, \cdots, x_{n-1}\right)=p\left(x_{0 \alpha}, \cdots, x_{(n-1) \alpha}\right) .
$$

Note that $\alpha \in G(p)$ if and only if $p=p^{\alpha}$.

Let $P_{n+1}(i)$ denote the set of all $(n+1)$-ary polynomials $p$ which can be represented in the form

$$
p=q\left(x_{0}, \cdots, x_{i-1}, x_{i+1}, \cdots, x_{n}\right) x_{i}
$$

for some $q \in P_{n}$. It follows from Lemma 4 that $P_{n+1}(i) \subseteq P_{n+1}$, and that $q$ is uniquely determined by $p$. If $p \in P_{n+1}(i)$ the variable $x_{i}$ is said to split in $p$.

Lemma 9. If $x_{i}$ splits in $p \in P_{n+1}$, and $\alpha \in G(p)$, then $x_{i \alpha}$ also splits in $p$.

Proof. Obvious from (22) and (23).

Lemma 10. Let $p \in P_{n}$. Then $p=x_{0} \cdot \cdots \cdot x_{n-1}$ if and only if all $x_{i}$ split in $p$.

Proof. It is obvious that if $p=x_{0} \cdots \cdots \cdot x_{n-1}$, then all $x_{i}$ split in $p$. Conversely, assume that all $x_{i}$ split in $p$. Then, for some $q \in P_{n-1}$, $q\left(x_{0}, \cdots, x_{i-1}, x_{i+1}, \cdots, x_{n-1}\right) x_{i}=p$, and so

$$
\begin{aligned}
p\left(x_{0}, \cdots, x_{i} y_{i}, \cdots, x_{n-1}\right) & =q\left(x_{0}, \cdots, x_{i-1}, x_{i+1}, \cdots, x_{n-1}\right) x_{i} y_{i} \\
& =p\left(x_{0}, \cdots, x_{i}, \cdots, x_{n-1}\right) y_{i} .
\end{aligned}
$$

Now compute using (24):

$$
\begin{aligned}
p\left(x_{0} y_{0}, \cdots, x_{i} y_{i}, \cdots, x_{n-1} y_{n-1}\right) & =p\left(x_{0}, \cdots, x_{n-1}\right) y_{0} \cdots y_{n-1} \\
& =p\left(y_{0}, \cdots, y_{n-1}\right) x_{0} \cdots \cdot x_{n-1}
\end{aligned}
$$

Setting $y_{0}=\cdots=y_{n-1}=y$ we get

$$
p\left(x_{0}, \cdots, x_{n-1}\right) y=y \cdot x_{0} \cdot \cdots \cdot x_{n-1} .
$$


And so

$$
\begin{aligned}
p\left(x_{0}, \cdots, x_{n-1}\right) & =p\left(x_{0}, \cdots, x_{n-1}\right) \cdot p\left(x_{0}, \cdots, x_{n-1}\right) \\
& ={ }^{(26)} p\left(x_{0}, \cdots, x_{n-1}\right) \cdot x_{0} \cdot \cdots \cdot x_{n-1} \\
& =x_{0} \cdot \cdots \cdot x_{n-1} \cdot x_{0} \cdot \cdots \cdot x_{n-1} \\
& =x_{0} \cdot \cdots \cdot x_{n-1},
\end{aligned}
$$

which was to be proved.

4. The inequality $p_{n+1} \geqq 2 p_{n}+1$. In this and the next section let $\mathfrak{A}$ be an algebra satisfying the conditions of Theorem 2 , and let $n$ be a fixed integer with $p_{n}(\mathfrak{U}) \neq 1$. Now we proceed to proving the inequality given in the title of the section.

For $p \in P_{n}$ let $R(p)$ denote the set of all polynomials of the form $p M_{i}$, or $p S$. By Lemmas 3 and $4, R(p) \subseteq P_{n+1}$. If $p=x_{0} \cdot \cdots \cdot x_{n-1}$, then $|R(p)|=1$, in fact, $R(p)=\left\{x_{0} \cdot \cdots \cdot x_{n-1} \cdot x_{n}\right\}$.

Lemma 11. If $p \neq x_{0} \cdot \cdots \cdot x_{n-1}$, then $|R(p)| \geqq 2$.

Proof. Let $|R(p)|=1$. Then $p M_{0}=p M_{1}=\cdots=p M_{n-1}$. Thus by Lemma 7 any pair of variables can be replaced by their products. Applying this a number of times we get

$$
p=p\left(x_{0} \cdot \cdots \cdot x_{n-1}, \cdots, x_{0} \cdot \cdots \cdot x_{n-1}\right)=x_{0} \cdot \cdots \cdot x_{n-1},
$$

as claimed.

Lemma 12. Let $p, q \in P_{n}, p \neq q$. Then $R(p)$ and $R(q)$ are disjoint.

Proof. By Lemmas 3, 4, 5, and 6 .

By Lemmas 11 and 12,

$$
p_{n+1} \geqq\left|\bigcup\left(R(p) \mid p \in P_{n}\right)\right| \geqq 2 p_{n}-1 \text {. }
$$

Lemma 13. If $p_{n+1}<2 p_{n}+1$, then $|R(p)|=2$ for all $p \in P_{n}, p \neq$ $x_{0} \cdot \cdots \cdot x_{n-1}$.

Proof. It follows from (27) that $p_{n+1}=2 p_{n}$ or $p_{n+1}=2 p_{n}-1$, and so $|R(p)|=2$ for all $p \in P_{n}, p \neq x_{0} \cdots x_{n-1}$, with at most one exception. Let $p$ be this exception; then $|R(p)|=3$.

Partition $\{0, \cdots, n-1\}$ into (at most) three classes, $X_{0}, X_{1}, X_{2}$ as follows:

$i, j \in X_{a}$ for some $a$, if $p M_{i}=p M_{j}$; furthermore, if $i \in X_{2}$, then $p M_{i}=p S$. 
Since $|R(p)|=3,\left|X_{0}\right| \neq 0,\left|X_{1}\right| \neq 0$, but $X_{2}$ could be empty. Note that by Lemma $7 i, j \in X_{a}$, if and only if $x_{i}$ and $x_{j}$ can be substituted by $x_{i} x_{j}$; hence if $i \in X_{a}, j \in X_{b}, a \neq b$, then this cannot hold for $x_{i}$ and $x_{j}$.

Now we distinguish some cases:

Case 1. For some $a\left|X_{a}\right| \geqq 2$. Then choose $i, j \in X_{a}, i \neq j, k \in X_{b}$, $a \neq b$. To simplify the computation let $0,1 \in X_{a}, 2 \in X_{b}$. Let $\tau$ be the transposition $(0,2)$. We claim that $p \neq p^{\tau}$. Indeed, if $p=p^{\tau}$, then

$$
\begin{aligned}
p\left(x_{0}, x_{1}, x_{2}, \cdots\right) & =p\left(x_{0} x_{1}, x_{0} x_{1}, x_{2}, \cdots\right) \\
& =p\left(x_{2}, x_{0} x_{1}, x_{0} x_{1}, \cdots\right) \\
& =p\left(x_{0} x_{1} x_{2}, x_{0} x_{1} x_{2}, x_{0} x_{1}, \cdots\right) \\
& =p\left(x_{0} x_{1}, x_{0} x_{1} x_{2}, x_{0} x_{1} x_{2}, \cdots\right) \\
& =p\left(x_{0} x_{1} x_{2}, x_{0} x_{1} x_{2}, x_{0} x_{1} x_{2}, x_{3}, \cdots\right) .
\end{aligned}
$$

Similarly,

$$
p\left(x_{0} x_{2}, x_{1}, x_{0} x_{2}, \cdots\right)=p\left(x_{0} x_{1} x_{2}, x_{0} x_{1} x_{2}, x_{0} x_{1} x_{2}, \cdots\right),
$$

and so $p\left(x_{0}, x_{1}, x_{2}, \cdots\right)=p\left(x_{0} x_{2}, x_{1}, x_{0} x_{2}, \cdots\right)$, contradicting $0 \in X_{a}, 2 \in X_{b}$, $a \neq b$.

Thus $p \neq p^{\tau}$. Since $|R(p)|=\left|R\left(p^{\tau}\right)\right|$, we get a contradiction with the uniqueness of $p$.

Case 2. $\left|X_{a}\right| \leqq 1$ for $a=0,1,2$, and $X_{2} \neq \varnothing$. Since $\left|X_{2}\right|=n$ is impossible, let $\left|X_{0}\right| \neq 0$, and take $\mathrm{i} \in X_{0}, j \in X_{2}, \tau=(i, j)$. Then $p=p^{\tau}$ would imply $p M_{i}=p S$; since $p M_{j}=p S$, we obtain $p M_{i}=p M_{j}$, contradicting the definition of $X_{2}$, and $i \notin X_{2}$. Hence $p \neq p^{\tau},\left|R\left(p^{\tau}\right)\right|=|R(p)|=$ 3 , a contradiction.

Case 3. $\left|X_{0}\right|=\left|X_{1}\right|=1$, and $X_{2}=\varnothing$. Thus in this case $n=2$, and $p M_{0}, p M_{1}, p S$ are all distinct. Take $\tau=(0,1)$. If $p \neq p^{\tau}$, then $|R(p)|=\left|R\left(p^{\tau}\right)\right|=3$, a contradiction. Hence, $p\left(x_{0}, x_{1}\right)=p\left(x_{1}, x_{0}\right)$. Let us denote $p\left(x_{0}, x_{1}\right)$ by $x_{0}+x_{1}$. Then - and + satisfy the requirements of Lemma 2. Since $p_{3} \leqq 2 p_{2}$, all essentially ternany with at most one exception are accounted for by $\mathrm{U}\left(R(t) \mid t \in P_{2}\right)$. But the seven polynomials listed in (10) can belong to no $R(t)$ excepting $R(+)$. (The verification of this statement is tedious but straightforward.) Hence either $|R(+)|>3$, or there are at least five essentially ternary polynomials outside of $\bigcup\left(R(t) \mid t \in P_{2}\right)$, contradicting the assumptions.

Cases 1-3 exhaust all possibilities, thus completing the proof of Lemma 13.

Lemma 14 . If $|R(p)| \leqq 2$ for all $p \in P_{n}$, then all $p \in P_{n}, p \neq x_{0} \cdot \cdots$ 
- $x_{n-1}$, have a unique representation in the form (8), where $\circ$ is an essentially binary noncommutative polynomial satisfying (9); this polynomial o is uniquely determined by $p$.

Proof. Let $p \in P_{n}, p \neq x_{0} \cdot \cdots \cdot x_{n-1}$, and so $|R(p)|=2$. Thus $\{0, \cdots, n-1\}$ splits into two nonvoid sets $X_{0}, X_{1}$ such that for $i, j \in X_{0}$, $p M_{i}=p M_{j}$, and for $i \in X_{1}, p M_{i}=p S$. Thus by Lemma 7 , for $i \in X_{0}$, $x_{i}$ can be replaced by the product of all $x_{j}, j \in X_{0}$, for $i \in X_{1}, x_{i}$ can be replaced by the product of all $x_{j}, j \in X_{1}$, and all these variables split in $p x_{n}$. Define 。 by

$$
x \circ y=p\left(z_{0}, \cdots, z_{n-1}\right),
$$

where $z_{i}=x$ for $i \in X_{1}, z_{i}=y$ for $i \in X_{0}$. Setting $X_{1}=\left\{i_{0}, \cdots, i_{k}\right\}$, (8) gives $p$. The uniquness of $\circ$, and (9) follow from the fact that the $x_{i}, i \in X_{0}$ do not split, while the $x_{i}, i \in X_{1}$ do in $p x_{n}$.

Now we are ready to complete the proof of the inequality. If $p_{n+1} \geqq 2 p_{n}+1$ does not hold, then $p_{n+1} \leqq 2 p_{n}$, hence by Lemma 13, $\mid R(p)) \leqq 2$ for all $p \in P_{n}$. By Lemma 14, (8) gives a unique representation for every $p \in P_{n}, p \neq x_{0} \cdot \cdots \cdot x_{n-1}$, and Lemma 1 stated that every such polynomial is essentially $n$-ary. Let $k$ denote the number of essentially binary polynomials satisfying the requirements of Lemma 1. Then it follows from what has been stated above that

$$
p_{n}=k\left(2^{n}-2\right)+1 \text {. }
$$

Again applying Lemma 1, we obtain the inequality

$$
p_{n+1} \geqq k\left(2^{n+1}-2\right)+1 \text {. }
$$

Hence

$$
k\left(2^{n+1}-2\right)+1 \leqq p_{n+1} \leqq 2 p_{n}=2 k\left(2^{n}-2\right)+2,
$$

yielding $2 k \leqq 1$, that is $k=0$. Therefore $p_{1}=1$, contrary to assumption. This completes the proof of the inequality.

5. The inequality $p_{n+1} \geqq p_{n}+n+2$. Recall that $P_{n+1}(i)$ is the set of all polynomials with representation (23). By Lemma 4, $\left|P_{n+1}(i)\right|=$ $p_{n}$. By Lemma 10, $\cap\left(P_{n+1}(i) \mid 0 \leqq i \leqq n\right)=\left\{x_{0} \cdot \cdots \cdot x_{n}\right\}$, hence we can choose

$$
p\left(x_{0}, \cdots, x_{n-1}\right) x_{n} \in P_{n+1}(n)-P_{n+1}(n-1),
$$

that is, $p \in P_{n}$ can be chosen such that $x_{n-1}$ does not split in $p x_{n}$. Define:

$$
q=p\left(x_{0}, \cdots, x_{n-1} x_{n}\right)
$$


Lemma 15. Neither $x_{n-1}$ nor $x_{n}$ splits in $q$.

Proof. $x_{n-1}$ and $x_{n}$ are symmetric in $q$, therefore it suffices to prove that $x_{n}$ does not split in $q$. Let us assume that $x_{n}$ splits in $q$, that is

$$
q=r\left(x_{0}, \cdots, x_{n-1}\right) x_{n} .
$$

Now substitute $x_{n}=x_{n-1}$ in (28) and (29); we obtain

$$
p\left(x_{0}, \cdots, x_{n-1}\right)=r\left(x_{0}, \cdots, x_{n-1}\right) x_{n-1} .
$$

Substituting $x_{n-1} x_{n}$ for $x_{n-1}$, and comparing the result with (28) and (29) we obtain

$$
r\left(x_{0}, \cdots, x_{n-1} x_{n}\right) x_{n-1} x_{n}=r\left(x_{0}, \cdots, x_{n-1}\right) x_{n} .
$$

Thus

$$
p\left(x_{0}, \cdots, x_{n-1}\right) x_{n}={ }^{(30)} r\left(x_{0}, \cdots, x_{n-1}\right) x_{n-1} x_{n}={ }^{(31)} r\left(x_{0}, \cdots, x_{n-1} x_{n}\right) x_{n-1} x_{n} .
$$

This formula shows that $p x_{n}$ is symmetric in $x_{n-1}$ and $x_{n}$, contradicting the assumption that $x_{n-1}$ does not split in $p x_{n}$.

Now we start proving the inequality. Let $s$ denote the number of variables that split in $q$.

Case 1. $s \geqq 2$. Let $Q$ denote the set of all polynomials arising from $q$ by permuting $x_{0}, \cdots, x_{n-1}$. Note that $P_{n+1}(n) \cap Q=\varnothing$. Of the $n$ ! permutations (by Lemma 9 ) at most $(n-s) ! \cdot s$ ! belong to $G(q)$, hence by Lemma 8 ,

$$
\begin{aligned}
|Q| \geqq \frac{n !}{(n-s) ! \cdot s !}=\left(\begin{array}{l}
n \\
s
\end{array}\right) \geqq\left(\begin{array}{l}
n \\
2
\end{array}\right) \geqq n+2, \\
\quad \text { for } n \geqq 4, \text { and } s<n-1 .
\end{aligned}
$$

Thus, if $n \geqq 4$, and $s<n-1$, then

$$
\left|P_{n+1}\right| \geqq\left|P_{n+1}(n) \cup Q\right| \geqq\left|P_{n+1}(n)\right|+|Q| \geqq P_{n}+n+2 \text {. }
$$

Let $n=3 ; s \geqq 2$, hence $s=2\left(s=3\right.$ implies that $\left.p=x_{0} \cdot x_{1} \cdot x_{2}\right)$. Thus $x_{0}$ and $x_{1}$ split in $q\left(x_{0}, x_{1}, x_{2}, x_{3}\right)=p\left(x_{0}, x_{1}, x_{2} x_{3}\right)$, and so $q=p\left(x_{0} x_{1}\right.$, $\left.x_{0} x_{1}, x_{2} x_{3}\right)$. Set $x \circ y=p(x, x, y)$. Then $\circ$ satisfies (9) and so (8) will produce seven essentially 4 -ary polynomials in which $x_{3}$ does not split. Thus $p_{4} \geqq p_{3}+7 \geqq p_{3}+3+2$. Finally, if $n \geqq 4$, and $s=n-1$, then as in the previous case we set $x \circ y=p(x, \cdots, x, y)$ and apply (8) to get $p_{n+1} \geqq p_{n}+2^{n-1} \geqq p_{n}+n+2$.

Case 2. $s=1$. Let $x_{0}$ be the variable that splits in $q$. Let $Q$ 
be defined as in Case 1. Since by Lemma 9 one variable (the one that splits) has to be kept fixed by any $\alpha \in G(q)$ we get that at most $(n-1)$ ! permutations of $\{0, \cdots, n-1\}$ belong to $G(q)$, and therefore we get at least $n$ polynomials from $q$ by permuting $x_{0}, \cdots, x_{n-1}$. We get exactly $n$, if every permutation not moving 0 belongs to $G(q)$. Thus if we get exactly $n$, all transpositions $(i, n) \in G(p), i \neq 0$. But then

$$
\begin{aligned}
q\left(x_{0}, x_{1},\right. & \left.\cdots, x_{n}\right) \\
& =p\left(x_{0}, x_{1}, \cdots, x_{n-1} x_{n}\right) \\
& =p\left(x_{0}, x_{n-1} x_{n}, \cdots, x_{1} x_{n-1} x_{n}\right) \\
& =p\left(x_{0}, x_{1} x_{n-1} x_{n}, \cdots, x_{1} x_{n-1} x_{n}\right)=\cdots \\
& =p\left(x_{0}, x_{1} x_{2} \cdots x_{n}, x_{1} x_{2} \cdots \cdot x_{n}, \cdots, x_{1} x_{2} \cdot \cdots \cdot x_{n}\right) .
\end{aligned}
$$

Also, since $x_{0}$ splits in $q$ :

$$
q\left(x_{0}, \cdots, x_{n}\right)=r\left(x_{1}, \cdots, x_{n}\right) \cdot x_{0} .
$$

From (32) and (33) we obtain,

$$
\begin{aligned}
q\left(x_{0}, \cdots, x_{n}\right) & =r\left(x_{1} \cdot \cdots \cdot x_{n}, \cdots, x_{1} \cdot \cdots x_{n}\right) \cdot x_{0} \\
& =x_{0} \cdot x_{1} \cdots \cdot x_{n} .
\end{aligned}
$$

Thus $p=x_{0} \cdot \cdots \cdot x_{n-1}$, contrary to assumption. Thus we cannot get exactly $n$, hence we get at least $2 n$, and so

$$
p_{n+1} \geqq p_{n}+2 n \geqq p_{n}+n+2,
$$

because $n \geqq 2$.

Case 3. Cases 1 and 2 do not apply to any

$$
p x_{n} \in P_{n+1}(n)-P_{n+1}(n-1) \text {. }
$$

Firstly we claim that $p_{n-1}=1$. Indeed, if $p_{n-1} \neq 1$, then let $r$ be an essentially $(n-1)$-ary polynomial different from $x_{0} \cdots \cdots x_{n-2}$. Then some $x_{i}$, say $x_{0}$ does not split in $r \cdot x_{n-1} \cdot x_{n}$, hence by permuting the variables we get a $p x_{n} \in P_{n+1}(n)-P_{n+1}(n-1)$ such that some $x_{i}$ splits in $p\left(x_{0}, \cdots, x_{n-1} x_{n}\right)$.

Now choose an arbitrary $p x_{n} \in P_{n+1}(n)-P_{n+1}(n-1)$ and take $q=$ $p\left(x_{0}, \cdots, x_{n-1} x_{n}\right)$. Note that in $q$ the pair $\left\{x_{n-1}, x_{n}\right\}$ is the only one which can be substituted by their product, because if $\left\{x_{i}, x_{j}\right\}$ is any other such pair then by setting $x_{n-1}=x_{n}, x_{i}=x_{j}$ we would get an $(n-1)$-ary polynomial different from $x_{0} \cdot \cdots \cdot x_{n-2}$, in contradiction with $p_{n-1}=1$. Hence for every $\alpha \in G(q),(n-1) \alpha=n-1$ and $n \alpha=n$, or $(n-1) \alpha=n, n \alpha=n-1$. Thus $|G(q)| \leqq(n-1) ! 2$ !, and so we get at least $\left(\begin{array}{c}n+1 \\ 2\end{array}\right) \geqq n+2$ polynomials by permuting the variables of $q$, 
none of them in $P_{n+1}(n)$, provided $\mathrm{n} \geqq 3$.

If $n=2$, then $p\left(x_{0}, x_{1} x_{2}\right)$ yields three polynomials in which no variable splits; $\left|P_{3}(2)\right|=p_{2}$ and we can choose a $t \in P_{3}(1)-P_{3}(2)$, obtaining $p_{2}+4$ polynomials. This completes the proof of the inequality.

6. The nonassociative case. In this section let $\mathfrak{A}$ be an idempotent algebra, and - a binary commutative and nonassociative polynomial. The following lemma is due to J. Dudek [1]:

Lemma 16. Let $n>2$ and let $f_{n}$ denote the polynomial

$$
\left(\cdots\left(\left(x_{0} x_{1}\right) x_{2}\right) \cdots\right) x_{n-1} \text {. }
$$

Let $\tau$ be the transposition $(i, i+1)$, where $i \neq 0$, and let $\sigma$ denote the cyclic permutation $(0,1, \cdots, n-1)$. Then $f_{n} \neq f_{n}^{\tau}$, and the polynomials $f_{n}, f_{n}^{\sigma}, f_{n}^{\sigma^{2}}, \cdots, f_{n}^{\sigma^{n-1}}$ are all distinct.

Now we prove the inequality $p_{n+1} \geqq p_{n}+(n-1)$. Observe that Lemma 3 applies, hence $\left|P_{n} M_{n-1}\right|=p_{n}$ and $P_{n} M_{n-1} \subseteq P_{n+1}$. We claim that $f_{n+1}, f_{n+1}^{\sigma}, \cdots, f_{n+1}^{\sigma n-2} \notin P_{n} M_{n-1}$. Indeed, let $f_{n}^{\sigma k} \in P_{n} M_{n-1}$. Then

$$
\left(\cdots\left(\left(\left(\cdots\left(x_{k} x_{k+1}\right) \cdots\right) x_{n}\right) x_{0}\right) \cdots\right) x_{k-1}=p\left(x_{0}, \cdots, x_{n-1} x_{n}\right) \text {. }
$$

Since $x_{n-1}$ and $x_{n}$ are symmetric in the right hand side, we get that $f_{n+1}$ is invariant under some $\tau=(i, i+1), i \neq 0$, contrary to Lemma 16. Thus we have found $p_{n}+(n-1)$ essentially $(n+1)$-ary polynomials, completing the proof.

\section{REFERENCES}

1. J. Dudek, The number of algebraic operations in an idempotent groupoid, Bull. Acad. Polon. Sci. Sér. Math. Phy. Astr. (to appear).

2. G. Grätzer, Universal algebra, The University Series in Higher Mathematics, D. Van Nostrand Co. Inc., Princeton, N. J., 1968.

3. G. Grätzer and R. Padmanabhan, On commutative, idempotent, and non-associative groupoids (to appear).

4. G. Grätzer and J. Płonka, A characterization of semilattices, Colloq. Math. (to appear).

5. - On the number of polynomials of a universal algebra II (to appear).

6. G. Grätzer, J. Płonka, and A. Sekanina, On the number of polynomials of a universal algebra I, Collog. Math. (to appear).

7. J. Płonka, On the number of independent elements in finite abstract algebras with two binary symmetrical operations, Colloq. Math. 19 (1868), 9-21.

8. - On a method of construction of abstract algebras, Fund. Math 61 (1967), 183-189.

9. K. Urbanik, On algebraic operations in idempotent algebras, Colloq. Math. 13 (1965), 129-157.

Received July 28, 1969, and in revised form November 4, 1969. The research of both authors was supported by the National Research Council of Canada.

The University of Manitoba 



\title{
PACIFIC JOURNAL OF MATHEMATICS
}

\author{
EDITORS
}

H. SAMELSON

Stanford University

Stanford, California 94305

J. DugunduI

Department of Mathematics

University of Southern California

Los Angeles, California 90007

RICHARD PIERCE

University of Washington

BASIL GORDON*

University of California

Seattle, Washington 98105

Los Angeles, California 90024

\section{ASSOCIATE EDITORS}

E. F. BeCKenbaCh

B. H. NeumanN

F. WOLF

K. YosHIDA

\section{SUPPORTING INSTITUTIONS}

\author{
UNIVERSITY OF BRITISH COLUMBIA \\ CALIFORNIA INSTITUTE OF TECHNOLOGY \\ UNIVERSITY OF CALIFORNIA \\ MONTANA STATE UNIVERSITY \\ UNIVERSITY OF NEVADA \\ NEW MEXICO STATE UNIVERSITY \\ OREGON STATE UNIVERSITY \\ UNIVERSITY OF OREGON \\ OSAKA UNIVERSITY \\ UNIVERSITY OF SOUTHERN CALIFORNIA
}

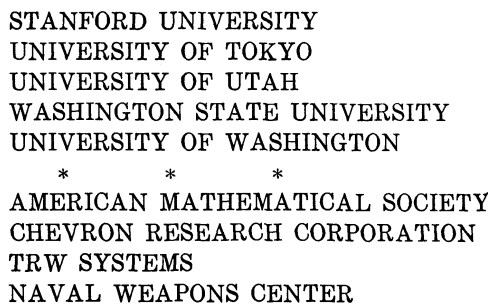

The Supporting Institutions listed above contribute to the cost of publication of this Journal, but they are not owners or publishers and have no responsibility for its content or policies.

Mathematical papers intended for publication in the Pacific Journal of Mathematics should be in typed form or offset-reproduced, double spaced with large margins. Underline Greek letters in red, German in green, and script in blue. The first paragraph or two must be capable of being used separately as a synopsis of the entire paper. It should not contain references to the bibliography. Manuscripts, in duplicate if possible, may be sent to any one of the four editors. Please classify according to the scheme of Math. Rev. 36, 1539-1546. All other communications to the editors should be addressed to the managing editor, Richard Arens, University of California, Los Angeles, California, 90024.

50 reprints are provided free for each article; additional copies may be obtained at cost in multiples of 50 .

The Pacific Journal of Mathematics is published monthly. Effective with Volume 16 the price per volume (3 numbers) is $\$ 8.00$; single issues, $\$ 3.00$. Special price for current issues to individual faculty members of supporting institutions and to individual members of the American Mathematical Society: $\$ 4.00$ per volume; single issues $\$ 1.50$. Back numbers are available.

Subscriptions, orders for back numbers, and changes of address should be sent to Pacific Journal of Mathematics, 103 Highland Boulevard, Berkeley, California, 94708.

PUBLISHED BY PACIFIC JOURNAL OF MATHEMATICS, A NON-PROFIT CORPORATION

Printed at Kokusai Bunken Insatsusha (International Academic Printing Co., Ltd.), 7-17, Fujimi 2-chome, Chiyoda-ku, Tokyo, Japan.

* Acting Managing Editor. 


\section{Pacific Journal of Mathematics}

\section{Vol. 32, No. 3 \\ March, 1970}

Shair Ahmad, Dynamical systems of characteristic $0^{+} \ldots \ldots \ldots \ldots \ldots$

Charles A. Akemann and Bernard Russo, Geometry of the unit sphere of a $C^{*}$-algebra and its dual............................ 575

Philip Bacon, The compactness of countably compact spaces ......... 587

Richard Blaine Barrar and Henry Loeb, On the continuity of the nonlinear Tschebyscheff operator ............................ 593

L. Carlitz, Factorization of a special polynomial over a finite field ....... 603

Joe Ebeling Cude, Compact integral domains .................... 615

Frank Rimi DeMeyer, On automorphisms of separable algebras. II . . . . . 621

James B. Derr, Generalized Sylow tower groups .................. 633

Raouf Doss, Some inclusions in multipliers ................... 643

Mary Rodriguez Embry, The numerical range of an operator........... 647

John Froese, Domain-perturbed problems for ordinary linear differential

operators..................................... 651

Zdeněk Frolík, Absolute Borel and Souslin sets ..................... 663

Ronald Owen Fulp, Tensor and torsion products of semigroups .......... 685

George Grätzer and J. Płonka, On the number of polynomials of an

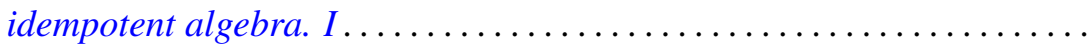

Newcomb Greenleaf and Walter Read, Positive holomorphic differentials on

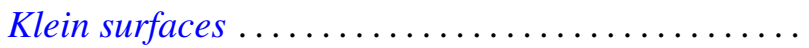

John Willard Heidel, Uniqueness, continuation, and nonoscillation for a second order nonlinear differential equation ................. 715

Leon A. Henkin, Extending Boolean operations................... 723

R. Hirshon, On hopfian groups .......................... 753

Melvin Hochster, Totally integrally closed rings and extremal spaces ..... 767

R. Mohanty and B. K. Ray, On the convergence of a trigonometric integral ..................................

Michael Rich, On a class of nodal algebras .................... 787

Emile B. Roth, Conjugate space representations of Banach spaces ........ 793

Rolf Schneider, On the projections of a convex polytope .............. 799

Bertram Manuel Schreiber, On the coset ring and strong Ditkin sets ...... 805

Edgar Lee Stout, Some remarks on varieties in polydiscs and bounded

holomorphic functions .........................

James Edward Ward, Two-groups and Jordan algebras . 